\title{
Activity-Dependent Control of Slow Synaptic Vesicle Endocytosis by Cyclin-Dependent Kinase 5
}

\author{
Gareth J. 0. Evans and Michael A. Cousin \\ Membrane Biology Group, Centre for Integrative Physiology, University of Edinburgh, Edinburgh EH8 9XD, United Kingdom
}

The stimulated dephosphorylation of the dephosphin group of endocytic proteins by calcineurin and their subsequent rephosphorylation by cyclin-dependent kinase 5 (cdk5) is required for synaptic vesicle (SV) retrieval in central nerve terminals. However, the specific endocytic pathway(s) controlled by these enzymes is unknown. To address this issue, we combined functional and morphological assays of endocytosis in primary neuronal cultures with pharmacological and molecular ablation of calcineurin and cdk5 activity. During strong stimulation, inhibition of calcineurin or cdk5 blocked uptake of the activity-dependent membrane marker FM1-43, but not the more hydrophilic FM2-10. However, FM2-10 uptake-measured poststimulation was sensitive to cdk5 and calcineurin inhibition, indicating that a slow form of endocytosis persists after termination of stimulation. In parallel EM studies, inhibition of cdk5 during strong stimulation greatly reduced horseradish peroxidase labeling of plasma membrane-derived nerve terminal endosomes, but not SVs. Furthermore, during mild stimulation, FM1-43 uptake was unaffected by cdk5 inhibition and the SV membrane was exclusively retrieved via a single SV route, suggesting that recruitment of the endosomal route of membrane retrieval is activity dependent. Thus, we propose that the calcineurin/cdk5-dependent phosphorylation cycle of the dephosphins specifically controls a slow endocytic pathway that proceeds via endosomal intermediates and is activated by strong physiological stimulation in central nerve terminals.

Key words: endocytosis; synaptic vesicle; nerve terminal; FM1-43; cdk5; dynamin

\section{Introduction}

Endocytosis of synaptic vesicles (SVs) after exocytosis is essential for the maintenance of neurotransmission in central neurons. The retrieval of the SV membrane from the nerve terminal plasma membrane can occur via at least two different routes. First, a rapid form of SV endocytosis exists where SVs are immediately available for release and do not pass through endosomal intermediates (Klingauf et al., 1998; Murthy and Stevens, 1998; Pyle et al., 2000; Stevens and Williams, 2000; Aravanis et al., 2003; Gandhi and Stevens, 2003). Second, a slower form exists that involves budding of SVs from endosomal intermediates where these intermediates have been generated either directly from large infoldings of the plasma membrane (Koenig and Ikeda, 1996; Takei et al., 1996; Richards et al., 2000, 2003) or by fusion of SVs retrieved by clathrin-dependent endocytosis (Heuser and Reese, 1973; Koenig and Ikeda, 1996). The rapid pathway (also known as fast endocytosis, reuse, or kiss-and-run) is thought to be the predominant SV recycling pathway during mild stimulation protocols (Aravanis et al., 2003; Gandhi and Stevens, 2003; Harata et al., 2006; Vanden Berghe and Klingauf, 2006). The slow

\footnotetext{
Received Sept. 1, 2006; revised Dec. 7, 2006; accepted Dec. 7, 2006.

This work was supported by The Wellcome Trust (GR070569). We thank Dr. Alan Prescott and John James (both at the University of Dundee, Dundee, UK) for their excellent technical assistance and Sander van den Heuvel and David Piston for the gift of plasmids.

Correspondence should be addressed to Michael A. Cousin, Membrane Biology Group, Centre for Integrative Physiology, George Square, University of Edinburgh, Edinburgh EH8 9XD, UK. E-mail: M.Cousin@ed.ac.uk.

G. J. 0. Evans' present address: Department of Biology (Area 3), University of York, P.0. Box 373, York Y010 5YW, UK.

DOI:10.1523/JNEUROSCI.3809-06.2007

Copyright $\odot 2007$ Society for Neuroscience $\quad$ 0270-6474/07/270401-11\$15.00/0
}

endocytosis pathway is proposed to be activated during more intense stimulation because of saturation of the fast endocytosis machinery, which is thought to have a limited capacity (Rizzoli and Betz, 2005). The fast and slow pathways are also thought to have separate functions, with fast endocytosis ensuring rapid refilling of the readily releasable pool, whereas slow endocytosis repopulates the total recycling SV pool both during and after high-intensity stimulation (Vanden Berghe and Klingauf, 2006).

The endocytosis of SVs in central nerve terminals is $\mathrm{Ca}^{2+}$. dependent and is stimulated by the same $\mathrm{Ca}^{2+}$ influx that triggers SV exocytosis (Marks and McMahon, 1998; Cousin, 2000). The $\mathrm{Ca}^{2+}$-dependent protein phosphatase calcineurin is a downstream $\mathrm{Ca}^{2+}$ effector for SV endocytosis, because its inhibition arrests the process in central nerve terminals (Marks and McMahon, 1998; Cousin et al., 2001; Smillie et al., 2005). At least eight nerve terminal substrates for calcineurin have been identified, which have been collectively called the dephosphins (Cousin and Robinson, 2001). The dephosphins are grouped together by two criteria: they are coordinately dephosphorylated by calcineurin on nerve terminal depolarization and they are all essential for SV endocytosis. After termination of nerve terminal stimulation, the dephosphins are rephosphorylated by their respective protein kinases. Cyclin-dependent kinase 5 (cdk5) rephosphorylates at least three of the dephosphins in vivo: the large GTPase dynamin I, the phosphatidylinositol kinase PIPKI $\gamma$, and the phosphatidylinositol phosphatase synaptojanin (Tan et al., 2003; Lee et al., 2004, 2005). Inhibition of cdk5 activity arrests multiple cycles of SV endocytosis in nerve terminals, demonstrating that the rephosphorylation of the dephosphins is equally essential as their dephosphorylation for the process (Tan et al., 2003). 
It is still unknown which endocytic pathway(s) the phosphorylation cycle of the dephosphins controls in nerve terminals. To determine this, we combined single-cell fluorescent imaging with quantitative electron microscopy. We found that $\mathrm{cdk} 5$-dependent protein rephosphorylation (and by extension the phosphorylation cycle of the dephosphins) directly controls slow, but not fast endocytosis in central nerve terminals.

\section{Materials and Methods}

Materials. Cyclosporin A (CsA), FK506, and roscovitine were from $\mathrm{CN}$ Biosciences (Nottingham, UK). Glutaraldehyde and osmium tetroxide were from Agar Scientific (Essex, UK). Penicillin/streptomycin, FM1-43, FM2-10, phosphate buffered salts, fetal calf serum, and Minimal Essential Medium were obtained from Invitrogen (Paisley, UK). Cdk5 plasmids were from Sander van den Heuvel (Harvard Medical School, Cambridge, MA) and DNA encoding mCerulean was from David Piston (Vanderbilt University, Nashville, TN). All other reagents were from Sigma (Poole, UK).

Fluorescence imaging of SV turnover. Primary cultures of cerebellar granule neurons were prepared from the cerebella of 7-d-old Sprague Dawley rat pups as described previously (Tan et al., 2003). SV turnover was monitored using a stimulation 2/stimulation 1 (S2/S1) protocol which determines responses from individual nerve terminals using an internal control (see Fig. 1A) (Smillie et al., 2005). Neurons were removed from culture medium and left for $10 \mathrm{~min}$ in incubation medium containing the following (in mM): $170 \mathrm{NaCl}, 3.5 \mathrm{KCl}, 0.4 \mathrm{KH}_{2} \mathrm{PO}_{4}, 20$ TES ( $N$-tris[hydroxy-methyl]-methyl-2-aminoethane-sulfonic acid), 5 $\mathrm{NaHCO}_{3}, 5$ glucose, $1.2 \mathrm{Na}_{2} \mathrm{SO}_{4}, 1.2 \mathrm{MgCl}_{2}, 1.3 \mathrm{CaCl}_{2}, \mathrm{pH}$ 7.4. Neurons were mounted in a Warner (Hamden, CT) imaging chamber (RC21BRFS) and the total recycling pool of SVs was loaded with either FM2-10 $(100 \mu \mathrm{M})$ or FM1-43 $(10 \mu \mathrm{M})$ by evoking SV recycling with a 50 mм KCl stimulus ( $50 \mathrm{~mm} \mathrm{NaCl}$ removed to maintain osmolarity) for 2 $\mathrm{min}$. This corresponds to $\mathrm{S} 1$ loading and results in labeling of fluorescent puncta corresponding to nerve terminals (see Fig. $1 \mathrm{~A}$ ). Cultures were immediately washed after termination of stimulation with incubation medium. After a $10 \mathrm{~min}$ rest period, accumulated dye was unloaded from nerve terminals using two sequential $30 \mathrm{~s} \mathrm{KCl}(50 \mathrm{~mm})$ challenges at 20 and $120 \mathrm{~s}$. This results in a decrease in fluorescence because of SV exocytosis (S1 unloading) (see Fig. 1 A). This $\mathrm{S} 1$ protocol provides an estimate of the total number of SVs turned over during maximal stimulation under control conditions $(\Delta \mathrm{S} 1)$. After a $20 \mathrm{~min}$ rest period, this protocol was repeated and the evoked responses in the same nerve terminals were examined (S2 loading and unloading) (see Fig. 1A). Thus, for any selected nerve terminal, the $\Delta \mathrm{S} 2$ response has a matched individual internal control $(\Delta \mathrm{S} 1)$. Nerve terminals were selected for analysis if they were able to be isolated visually from other nerve terminals and were able to load and unload dye during the $\mathrm{S} 1$ challenge. Results were analyzed by quantifying $\Delta \mathrm{S} 1$ (control) and $\Delta \mathrm{S} 2$ (test) for individual nerve terminals. These results were represented as either cumulative histograms $(\Delta S 2 / \Delta S 1$ for individual nerve terminals) or meaned data (average $\Delta S 2 / \Delta S 1$ for individual nerve terminals across different experiments). Where indicated, dye loading and unloading was evoked by action potential trains (100 $\mathrm{mA}, 1 \mathrm{~ms}$ pulse width) delivered using platinum wires embedded in the imaging chamber. Dye unloading was visualized using a Nikon (Tokyo, Japan) epifluorescence microscope (Diaphot-TMD) and $20 \times$ air objective at $480 \mathrm{~nm}$ excitation and $>510 \mathrm{~nm}$ emission. Fluorescent images were visualized using a Hamamatsu (Hamamatsu City, Japan) Orca-ER
CCD digital camera and offline imaging software (Compix Imaging Systems, Sewickley, PA). In all experiments, data from at least three independent experiments were collated each containing at least 70 nerve terminals (supplemental Table 1, available at www.jneurosci.org as supplemental material). Cultures were used routinely between 8 and $10 \mathrm{~d}$ in vitro.

Transfections and imaging of transfected neurons. Cultures were transfected with DNA using an established calcium phosphate precipitation protocol (Tan et al., 2003). Transfections were performed on cultures aged between 6 and $8 \mathrm{~d}$ in vitro and were routinely used $48 \mathrm{~h}$ later. Cultures were cotransfected with plasmids encoding mCerulean fluorescent protein [to mark transfected neurons (Rizzo et al., 2004)] and either WT or DN cdk5. Transfected neurons were located using excitation at $430 \mathrm{~nm}$ and a bandpass emission filter of $510-550 \mathrm{~nm}$. After dye loading, cultures were excited at $500 \mathrm{~nm}$ and emission was monitored between 510 and $550 \mathrm{~nm}$. All experiments using transfected cells were performed using a $40 \times$ oil-immersion objective (Zeiss, Oberkochen, Germany) on a Zeiss Axiovert 100-TV inverted fluorescence microscope. Excitation wavelengths were generated using a Till (Gräfelfing, Germany) Polychrome IV monochromator.

Electron microscopy. Cultures were processed for electron microscopy as described previously (Deak et al., 2004) with minor modifications. Cultures were removed into incubation media and after $10 \mathrm{~min}$ were given a prepulse of stimulation (either $10 \mathrm{~s}$ with $50 \mathrm{~mm} \mathrm{KCl}$ or 2 trains of 400 action potentials) to dephosphorylate the dephosphins. After a 10 min rest period cultures were stimulated with either $50 \mathrm{~mm} \mathrm{KCl}$ for $2 \mathrm{~min}$ or 200 action potentials in medium supplemented with horseradish peroxidase (HRP; $10 \mathrm{mg} / \mathrm{ml}$ ). Neurons were fixed in a $2 \%$ solution of glutaraldehyde in PBS for $30 \mathrm{~min}$ at $37^{\circ} \mathrm{C}$. After washing in $100 \mathrm{~mm}$ Tris, $\mathrm{pH}$ 7.4 , cultures were exposed to $0.1 \%$ diaminobenzidine and $0.2 \% \mathrm{H}_{2} \mathrm{O}_{2}$ in $100 \mathrm{~mm}$ Tris. On development of color, cultures were washed with 100 mu Tris and then postfixed with $1 \%$ osmium tetroxide for $30 \mathrm{~min}$. After washing, cultures were poststained with $2 \%$ uranyl acetate for $15 \mathrm{~min}$ 
A
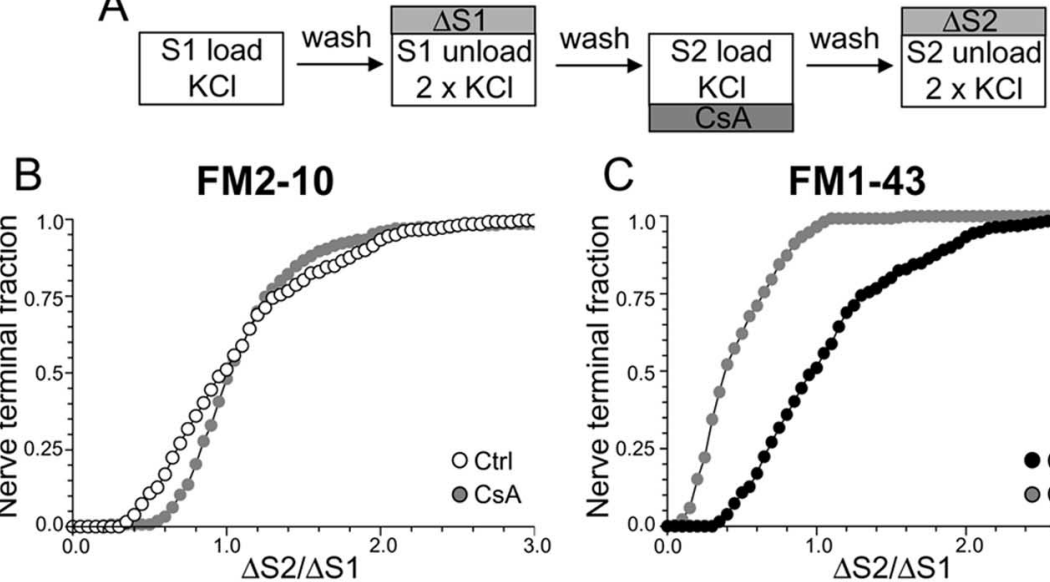

C

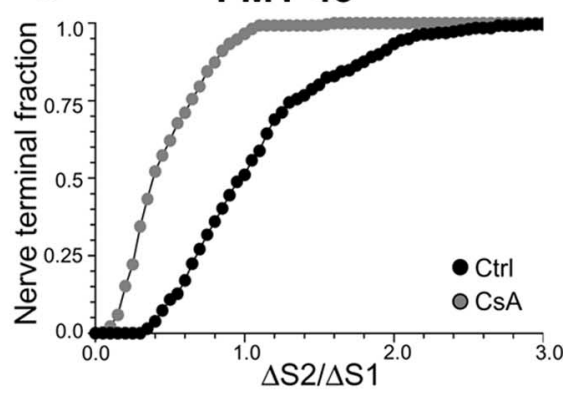

D
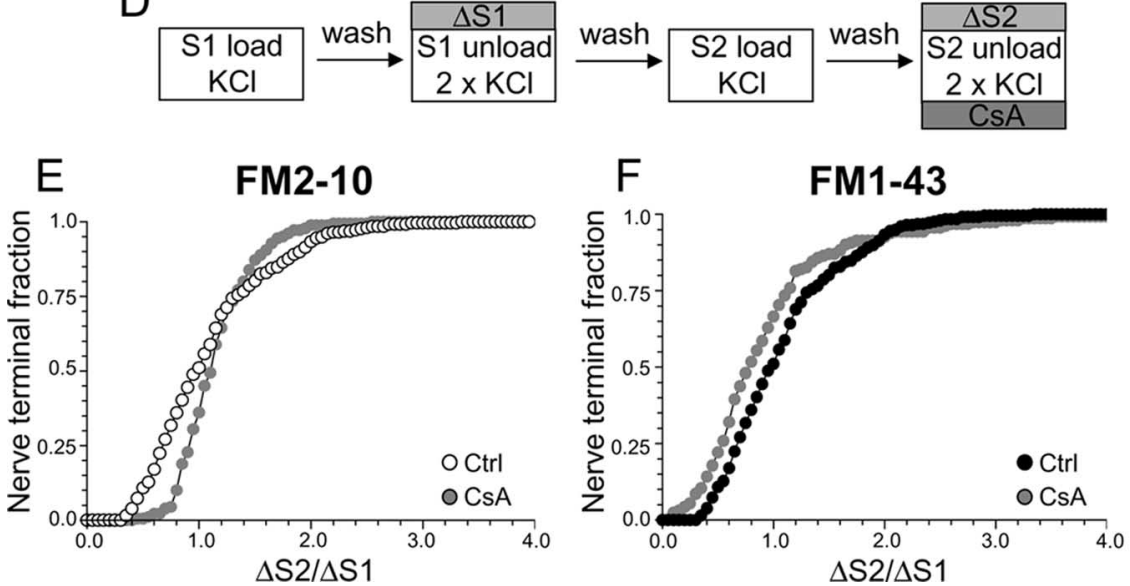

Figure 2. Antagonism of calcineurin inhibits uptake of FM1-43 but not FM2-10. $A$, Cultures were loaded and unloaded with either FM2-10 or FM1-43 using the same protocol as in Figure $1 A$. Where indicated, cultures were preincubated with $10 \mu \mathrm{M} C \mathrm{~A}$ for 10 min before and during S2 loading. $\boldsymbol{B}, \boldsymbol{C}$, Cumulative histograms of the effect of CsA on either FM2-10 (B) or FM1-43 (C) loading in individual nerve terminals $(\Delta \mathrm{S} 2 / \Delta \mathrm{S} 1, n=233, \mathrm{FM} 2-10$ control; $n=180, \mathrm{FM} 2-10 \mathrm{CsA} ; n=258, \mathrm{FM} 1-43$ control; $n=168, \mathrm{FM1}-43$ (SA). D , Cultures were loaded and unloaded with either FM2-10 or FM1-43 using the same protocol as in Figure $1 A$. Where indicated, cultures were preincubated with $10 \mu \mathrm{M}$ CsA for 10 min before and during $S 2$ unloading. $\boldsymbol{E}, \boldsymbol{F}$, Cumulative histograms of the effect of $C S A$ on either FM2-10 $(\boldsymbol{E})$ or FM1-43 $(\boldsymbol{F})$ unloading in individual nerve terminals ( $\Delta$ S2) $\Delta S 1, n=270, \mathrm{FM} 2-10 \mathrm{CsA} ; n=270, \mathrm{FM} 1-43 \mathrm{CsA})$. Open symbols, FM2-10; closed symbols, FM1-43; gray symbols, CsA. For all experiments, $n=3$.

then dehydrated using ethanol series and polypropylene oxide and embedded using Durcupan. Samples were sectioned, mounted on grids, and viewed using an FEI (Hillsboro, OR) Tecnai 12 transmission electron microscope. Intracellular structures that were $<100 \mathrm{~nm}$ in diameter were arbitrarily designated to be SVs, whereas larger structures were designated to be endosomes.

\section{Results}

An S2/S1 protocol was used to investigate the role of the phosphorylation status of the dephosphins in the control of different SV retrieval pathways in central nerve terminals (Fig. $1 A$ ). In this protocol, neuronal cultures were subjected to two rounds of styryl dye loading and unloading (S1 and S2). The extent of SV turnover during each stimulus was quantified by monitoring the extent of dye unloading from individual fluorescent puncta $(\Delta \mathrm{S} 1$ and $\Delta \mathrm{S} 2$ ). We used two similar dyes to label routes of SV membrane retrieval by endocytosis, FM2-10 and FM1-43. These dyes have almost identical properties; however, FM1-43 has a longer hydrocarbon tail than FM2-10, making it less prone to washout from membranes (Ryan et al., 1996; Richards et al., 2000). Thus FM1-43 should label endocytic structures that are generated

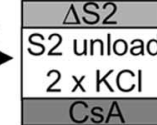

during and after its washout, whereas the more hydrophilic FM2-10 should only label those that are complete during stimulation (Richards et al., 2000; Virmani et al., 2003). When maximal SV turnover was stimulated at $\mathrm{S} 1$ and $\mathrm{S} 2$ with elevated $\mathrm{KCl}$ (50 mM), the mean $\Delta \mathrm{S} 2 / \Delta \mathrm{S} 1$ ratio for both FM2 -10 and FM1 -43 was $1.12 \pm 0.03$ and $1.08 \pm 0.04$, respectively, indicating reproducible labeling of SV pools between S1 and S2 for both FM2-10 and FM1-43 (Fig. 1C) (Smillie et al., 2005).

Using this protocol, we first determined the role of the calcium-dependent protein phosphatase calcineurin in different SV endocytosis pathways, because this enzyme dephosphorylates the dephosphins on nerve terminal stimulation (Liu et al., 1994; Marks and McMahon, 1998; Lee et al., 2004, 2005). Cultures were incubated with the calcineurin antagonist CsA at a concentration $(10 \mu \mathrm{M})$ that abolished the stimulation-dependent dephosphorylation of dynamin I in our culture system (supplemental Fig. 1, available at www. jneurosci.org as supplemental material). When CsA was added before and during the S2 loading stimulus (Fig. 2A) uptake of the less hydrophobic dye FM2-10 was unaffected by the presence of the drug (Fig. $2 B$ ) (average $\Delta \mathrm{S} 2 / \Delta \mathrm{S} 1$ : control, $1.12 \pm$ 0.03 ; CsA, $1.17 \pm 0.05 ; p=0.21$, two-way ANOVA, Bonferroni post hoc test) suggesting that SV retrieval activated during strong stimulation is not dependent on calcineurin activity (Smillie et al., 2005). However, when loading of the more hydrophobic FM1-43 was monitored using the identical stimulation protocol, a large reduction in dye uptake was observed, with almost every nerve terminal accumulating less FM1-43 in the presence of CsA than in its absence (Fig. 2C) (average $\Delta \mathrm{S} 2 /$ $\Delta \mathrm{S} 1$ : control, $1.08 \pm 0.03$; CsA, $0.46 \pm 0.02 ; p<0.001$, two-way ANOVA, Bonferroni post hoc test). Similar results were obtained using a different calcineurin antagonist (10 $\mu \mathrm{M}$ FK506) (data not shown). This disparity in loading between the two dyes indicates that FM1-43 may be labeling an additional endocytic pathway whose activation is dependent on calcineurin activity.

Styryl dye uptake is dependent on both the exocytosis and endocytosis of SVs during the loading stimulus (Smillie et al., 2005). Thus, to ensure that the observed effect of CsA was caused by inhibition of SV endocytosis and not exocytosis, cultures were incubated with CsA before and during the S2 unloading stimulus (Fig. 2D). This protocol will report any effect of the drug on SV exocytosis, independent of effects on SV endocytosis. CsA did not inhibit the unloading of either FM2-10 (Fig. 2E) (average $\Delta \mathrm{S} 2 /$ $\Delta \mathrm{S} 1$ : control, $1.12 \pm 0.03$; CsA, $1.14 \pm 0.02 ; p=0.13$, two-way ANOVA, Bonferroni post hoc test) or FM1-43 (Fig. $2 F$ ) (average $\Delta \mathrm{S} 2 / \Delta \mathrm{S} 1$ : control, $1.08 \pm 0.03 ; \mathrm{CsA}, 0.95 \pm 0.06 ; p=0.20$, two-way ANOVA, Bonferroni post hoc test), indicating that calcineurin activity is not required for $\mathrm{KCl}$-evoked SV exocytosis (Marks and McMahon, 1998; Cousin et al., 2001; Smillie et al., 2005). 
The disparity in dye loading observed after preincubation with CsA suggests that FM1-43, but not FM2-10, is labeling an endocytic pathway that is dependent on calcineurin activity. The most likely explanation is that this endocytic pathway persists after termination of stimulation, because FM2-10 would be washed out of these structures. To test this hypothesis, we delayed the washout of FM2-10 for 5 min after the termination of stimulation during dye loading at both S1 and S2 (Fig. 3A). This should allow FM2-10 to label the calcineurin-dependent endocytic pathway, thus mimicking FM1-43. Inhibition of calcineurin with CsA now reduced FM2-10 loading compared with control (Fig. $3 B$ ) (average $\Delta \mathrm{S} 2 / \Delta \mathrm{S} 1$ : control, $1.28 \pm 0.05$; CsA, $0.82 \pm 0.03 ; p<0.001$, two-way ANOVA, Bonferroni post hoc test). Thus, calcineurin activity controls the retrieval of SVs via an endocytic pathway that persists after termination of strong stimulation. We will call this form of retrieval slow endocytosis for the remainder of this manuscript.

\section{Protein rephosphorylation by cdk5 is essential for slow endocytosis}

Rephosphorylation of the dephosphins by cdk 5 after termination of stimulation is required to maintain multiple rounds of SV endocytosis (Cousin et al., 2001; Tan et al., 2003). Thus, we predicted that ablation of cdk5 activity should have the same effect on slow endocytosis as antagonism of calcineurin because they share a number of dephosphins as substrates (Tan et al., 2003; Lee et al., 2004, 2005). To test this hypothesis, we examined the effect of the selective cdk5 antagonist roscovitine on slow endocytosis. Roscovitine was used at a concentration $(50 \mu \mathrm{M})$ that abolished all cdk5 activity in our culture system, as determined by examining the phosphorylation status of its substrate dynamin I (supplemental Fig. 1, available at www.jneurosci.org as supplemental material). Addition of roscovitine during the S2 loading phase had no effect on FM1-43 loading (Fig. 4A,B). This was expected
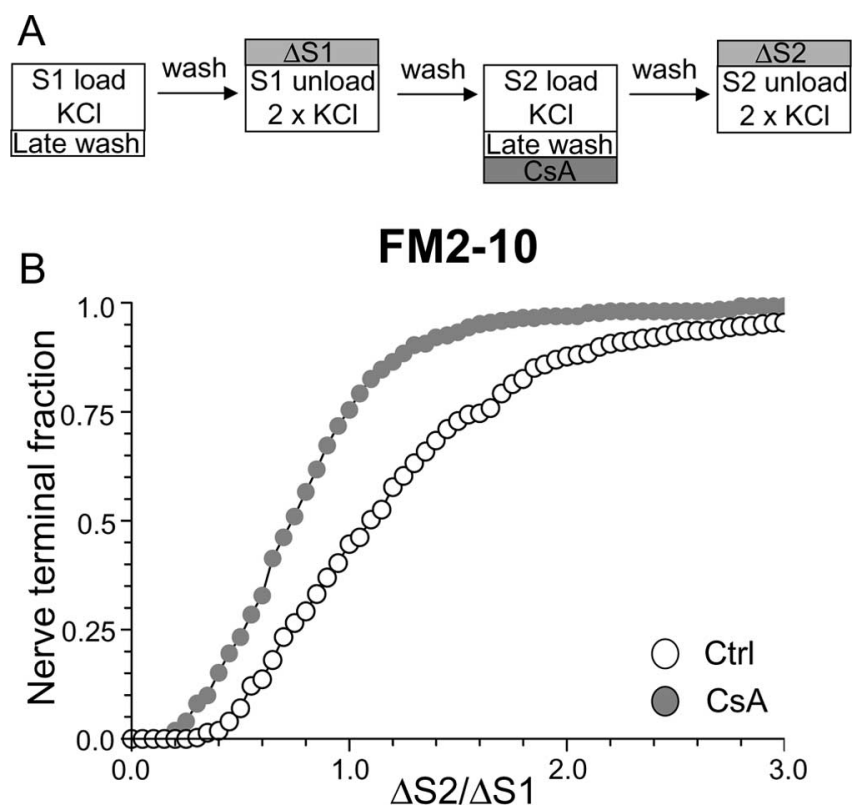

Figure 3. Delay of FM2-10 washout reveals calcineurin-dependent slow endocytosis. $\boldsymbol{A}$, FM2-10 was loaded using an identical protocol to Figure $1 A$ except wash of dye was delayed 5 min after termination of $\mathrm{KCl}$ stimulation at both $\mathrm{S} 1$ and $\mathrm{S} 2$. Where indicated, cultures were preincubated with $10 \mu \mathrm{M}$ CSA for 10 min before and during S2 loading. B, Cumulative histogram of the effect of CsA on dye loading in individual nerve terminals $(\Delta S 2 / \Delta S 1, n=270$ for both Ctrl and (sA). Open symbols, Control (Ctrl); gray symbols, (sA. For all experiments, $n=3$.
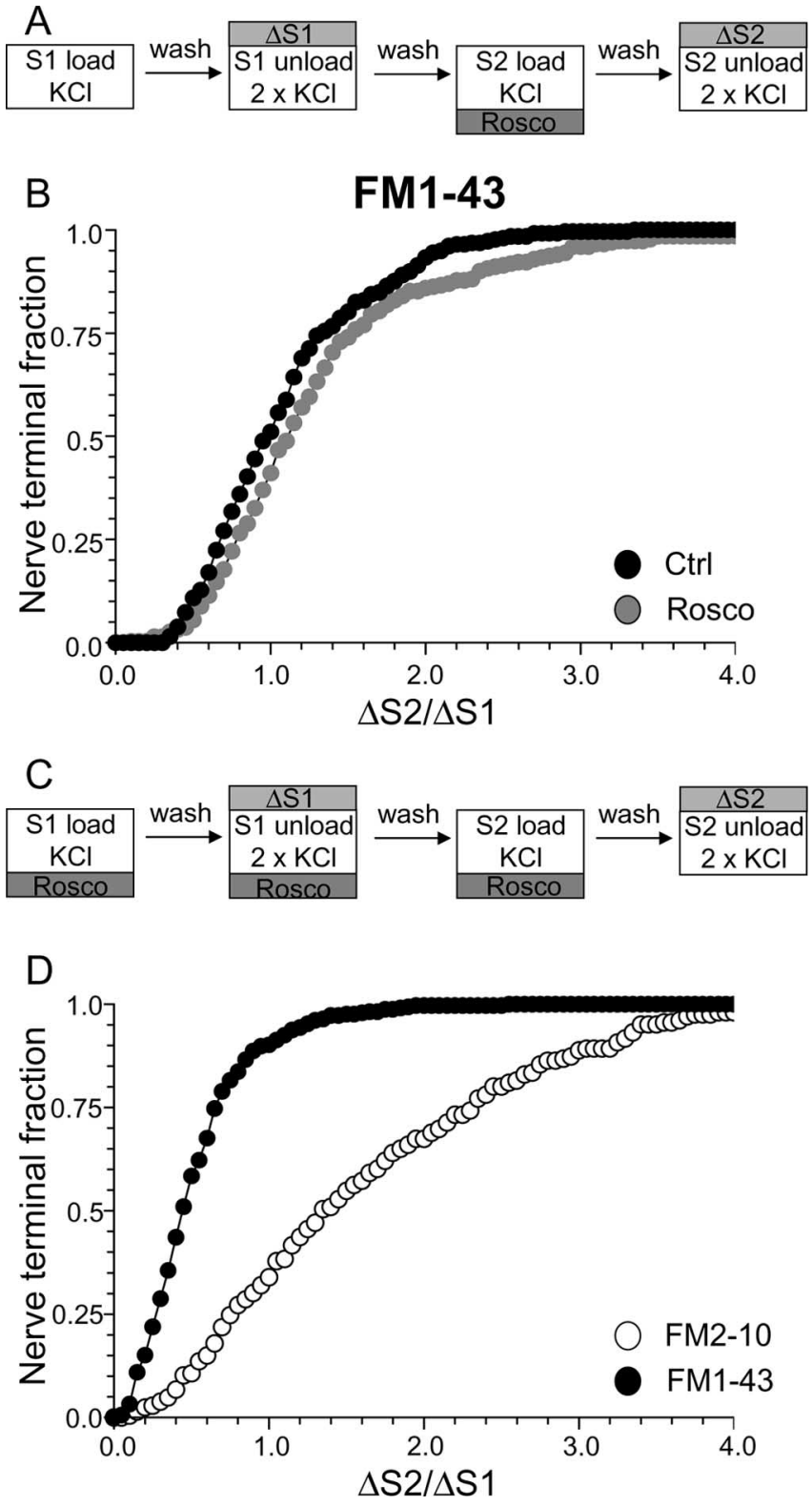

Figure 4. Antagonism of cdk5 inhibits uptake of FM1-43, but not FM2-10. A, Cultures were loaded and unloaded with FM1- 43 using the same protocol as in Figure $1 \mathrm{~A}$. Where indicated, cultures were preincubated with $50 \mu \mathrm{m}$ roscovitine (Rosco) for $10 \mathrm{~min}$ before and during $\mathrm{S2}$ loading. $\boldsymbol{B}$, Cumulative histogram of the effect of roscovitine on FM1- 43 loading in individual nerve terminals ( $\Delta S 2 / \Delta S 1, n=258$, Ctrl; $n=270$, Rosco). Closed symbols, Control (Ctrl); gray symbols, roscovitine. C, Cultures were loaded and unloaded with either FM2-10 and FM1-43 using an identical protocol to Figure $1 \mathrm{~A}$ except that cultures were preincubated with $50 \mu \mathrm{m}$ roscovitine for $10 \mathrm{~min}$ before and during all steps before and including $\$ 2$ loading. $\boldsymbol{D}$, Cumulative histogram of the effect of roscovitine on dye loading in individual nerve terminals ( $\Delta \mathrm{S} 2$ / $\Delta \mathrm{S} 1, n=209, \mathrm{FM} 2-10 ; n=337, \mathrm{FM} 1-43)$. Open symbols represent FM2-10 uptake whereas closed symbols represent FM1- 43 uptake both in roscovitine-treated cultures. For all experiments, $n=3$, apart from $n=4$ for FM1-43 data in $\boldsymbol{D}$.

because the dephosphins are phosphorylated in resting nerve terminals and inhibition of cdk5 will have no effect on SV endocytosis during the first round of SV turnover (Tan et al., 2003). This also indicates that (1) cdk5 does not participate in $\mathrm{KCl}$-evoked SV exocytosis in nerve terminals (Tan et al., 2003) and, importantly, (2) it eliminates any other cdk 5 substrates apart from the dephosphins from a role in SV endocytosis, because the phosphorylation of these putative substrates would have been inhibited by roscovitine using this protocol. 
To assess the specific role of cdk5-dependent rephosphorylation, we modified our protocol to have roscovitine present throughout the S1 stimulation period in addition to S2 loading (Fig. 4C). This permits the dephosphorylation of the dephosphins, but arrests their rephosphorylation (Tan et al., 2003), thus allowing a direct examination of their rephosphorylation by $\mathrm{cdk} 5$ in slow endocytosis. When this modified protocol was performed, FM1-43 uptake was extensively reduced in the majority of nerve terminals (Fig. $4 D$ ) (average $\Delta \mathrm{S} 2 / \Delta \mathrm{S} 1$ : control, $1.08 \pm$ 0.03; Rosco, $0.52 \pm 0.02 ; p<0.001$, two-way ANOVA, Bonferroni post hoc test). Thus, cdk5-dependent protein rephosphorylation is required for multiple cycles of SV endocytosis (Tan et al., 2003).

To determine whether the protein rephosphorylation by cdk 5 specifically controlled slow endocytosis, we performed the same protocol using FM2-10. A large disparity in FM1-43 and FM2-10 loading was observed in the presence of roscovitine (Fig. $4 D$ ) (average $\Delta \mathrm{S} 2 / \Delta \mathrm{S} 1$ : FM2-10 Rosco, $1.63 \pm 0.06$; FM1-43 Rosco, $0.52 \pm 0.02 ; p<0.001$, two-way ANOVA, Bonferroni post hoc test) indicating that cdk5 activity is required for slow endocytosis. FM2-10 loading was increased in the majority of roscovitine-treated nerve terminals. Because of this large facilitation of FM2-10 uptake, the delayed wash protocol used in Figure $2 D$ could not be used to confirm effects on slow endocytosis. Nevertheless, these results indicate that inhibition of protein rephosphorylation by cdk5 arrests a phase of endocytosis that is selectively labeled by FM1-43 and not FM2-10.

To ensure the effect of roscovitine on slow endocytosis was not caused by off-target effects on either related protein kinases or voltage-dependent calcium channels (Yan et al., 2002), we also examined the role of cdk5 by overexpression of dominantnegative (DN) and wild-type (WT) forms of the protein kinase. We showed previously that DN cdk5 only inhibits $~ 50 \%$ of the uptake of the dye FM4-64, which has a similar hydrophobicity to FM1-43 (Tan et al., 2003). However, if cdk5 selectively controls slow endocytosis, DN cdk5 should not inhibit loading of FM210. First, we determined the absolute amount of dye uptake for cells transfected with either WT or DN cdk5 (the S1/S2 protocol cannot be used in this instance because the mutant proteins are present throughout the experiment). Uptake of either FM2-10 or FM1-43 evoked by stimulation by $50 \mathrm{~mm} \mathrm{KCl}$ was not affected by overexpression of WT cdk5 (Fig. 5B). However, in nerve terminals overexpressing DN cdk5, FM1-43 uptake was reduced by approximately half (Fig. 5B). In contrast, FM2-10 uptake was unaffected by overexpression of the dominant-negative form of the protein kinase (Fig. 5B). This disparity again indicates a selective role for $\mathrm{cdk} 5$ in slow endocytosis and explains the incomplete inhibition of FM4-64 uptake by DN cdk5 observed in previous work (Tan et al., 2003).

To confirm such a role for cdk5, we performed a modified version of the delayed wash protocol using FM2-10 (Fig. 6A). In this instance, the washout of FM2-10 was only delayed at S2, which should result in an increase in FM2-10 loading if slow endocytosis is unaffected. When this experiment was performed, nerve terminals expressing DN cdk5 had a reduced uptake of FM2-10 in comparison to WT cdk5 transfected nerve terminals (Fig. $6 B$ ) (average $\Delta \mathrm{S} 2 / \Delta \mathrm{S} 1$ : WT cdk5, $2.00 \pm 0.14 ; \mathrm{DN} c d k 5$, $1.48 \pm 0.15 ; p<0.05$, two-way ANOVA, Bonferroni post hoc test) further confirming the essential role for cdk 5 in slow endocytosis. The observed effect of DN cdk5 is not attributable to effects on $\mathrm{KCl}$-evoked SV exocytosis, because expression of this construct does not affect this process in our cultures (Tan et al., 2003). Thus, either pharmacological or molecular ablation of cdk5 ac-
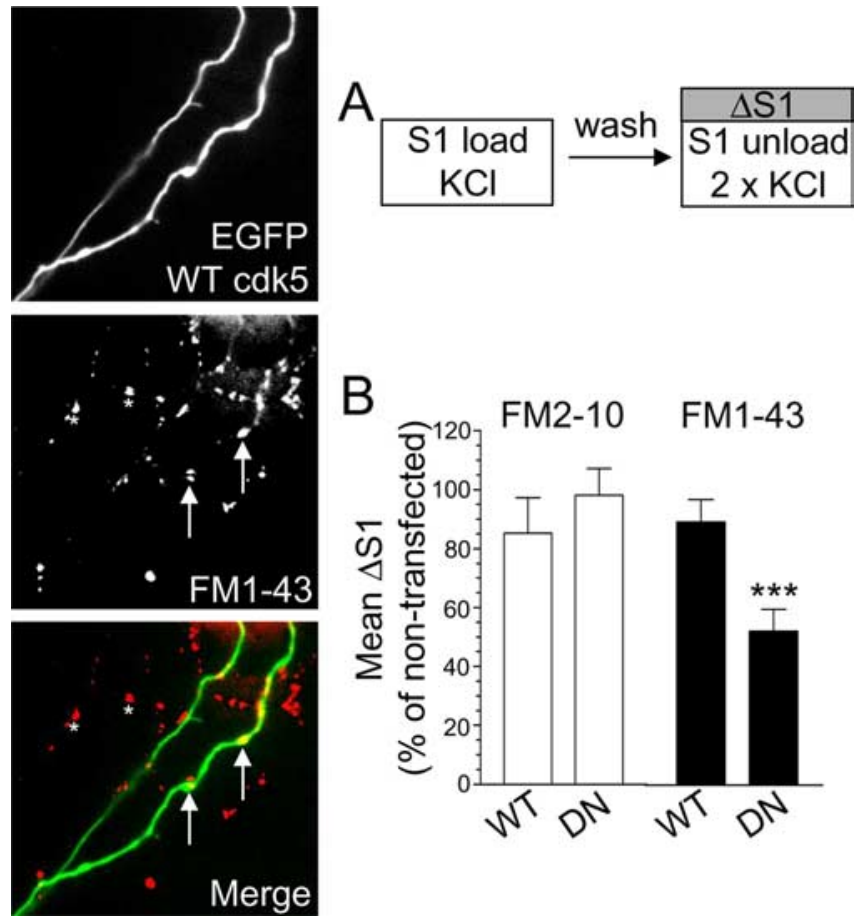

Figure 5. Overexpression of dominant-negative $\mathrm{cdk5}$ inhibits uptake of FM1-43, but not FM2-10. A, Cultures transfected with either WT or DN cdk5 were loaded and unloaded (S1) with either FM2-10 and FM1-43 using $50 \mathrm{~mm} \mathrm{KCl}$. Representative images show granule neuron cultures transfected with WT cdk5 and loaded with FM1-43. Merged image shows transfected neuron (green) and FM1-43-loaded nerve terminals (red). Arrows indicate transfected nerve terminals and asterisks indicate untransfected nerve terminals. Note the equal loading of both. $\boldsymbol{B}, \Delta \mathrm{S} 1$ response in transfected nerve terminals is displayed normalized to the $\mathrm{S1}$ from nontransfected nerve terminals in the same field of view. Open bars, FM2-10; closed bars, FM1-43 ( $n=31$, WT cdk5 FM2-10; $n=50$, DN cdk5 FM2-10; $n=63$, WT cdk5 FM1-43; $n=35$, DN cdk5 FM1-43; ${ }^{* * *} p<0.001$, Student's $t$ test). Error bars indicate SEM. For all experiments, $n=3$.

tivity inhibits a slow phase of SV endocytosis that persists after termination of strong stimulation.

We have shown an essential requirement for calcineurindependent dephosphorylation and cdk5-dependent rephosphorylation in slow endocytosis when SV turnover is stimulated by elevated $\mathrm{KCl}$. Next, we determined whether cdk5-dependent rephosphorylation was required for slow endocytosis that was evoked by physiological stimulation. To test this, we loaded dye using a protocol of 800 action potentials delivered at $80 \mathrm{~Hz}$ (Fig. $7 A$ ). This stimulation paradigm produces dye loading comparable with $\mathrm{KCl}$ stimulation in our cultures (G. J. O. Evans, unpublished observations). Dye was unloaded using elevated $\mathrm{KCl}$ as before. When this protocol was used, antagonism of protein rephosphorylation by cdk5 produced a large divergence in nerve terminal loading of FM1-43 and FM2-10 during actionpotential stimulation. Uptake of FM1-43 was strongly inhibited by roscovitine, whereas uptake of FM2-10 was unaffected compared with control (Fig. $7 B$ ) (average $\Delta \mathrm{S} 2 / \Delta \mathrm{S} 1$ : FM2-10 control, $1.09 \pm 0.03$; FM2-10 Rosco, $1.12 \pm 0.03 ; p=0.60$, two-way ANOVA, Bonferroni post hoc test) (Fig. 7C) (average $\Delta \mathrm{S} 2 / \Delta \mathrm{S} 1$ : FM1-43 control, $1.14 \pm 0.04$; FM1-43 Rosco, $0.66 \pm 0.03 ; p<$ 0.001, two-way ANOVA, Bonferroni post hoc test). Thus, cdk5dependent rephosphorylation is also essential for slow endocytosis when nerve terminals are subjected to strong physiological stimulation. 


\section{Inhibition of cdk 5 arrests endosome generation in} nerve terminals

Previous studies have suggested that slow endocytosis is morphologically distinct from fast endocytosis, with the SV membrane passing through endosomes that act as either sorting compartments or that are generated directly from the plasma membrane by bulk invagination (Koenig and Ikeda, 1996; Richards et al., 2000; de Lange et al., 2003). To identify a morphological correlate for $\mathrm{cdk} 5$-dependent slow endocytosis, we used HRP as a fluid phase endocytic marker to label all retrieving structures in our culture system during strong stimulation (Deak et al., 2004; Di Paolo et al., 2004). We performed a time course of HRP labeling of endocytic structures in the presence or absence of roscovitine (Fig. $8 \mathrm{~A}$ ). In this protocol, HRP was applied concurrent with the $\mathrm{KCl}$ stimulus for $2 \mathrm{~min}$ and then washed off at the termination of stimulation. Cultures were then allowed to repolarize for defined periods of time. When cultures were fixed during $\mathrm{KCl}$ stimulation, a large increase in the number of HRP-labeled endosomes was observed in control cultures (Fig. $8 \mathrm{~B}$ ). This was in contrast to roscovitine-treated nerve terminals, where the number of HRPlabeled endosomes was consistently reduced compared with control (Fig. $8 E$ ). This disparity in the number of labeled endosomes persisted throughout the duration of the time course (Fig. 9H). Thus, roscovitine greatly reduced the generation of endosomes from the plasma membrane during stimulation, suggesting cdk5dependent protein rephosphorylation is required for this endocytic pathway.

When the generation of HRP-labeled SVs was studied, there was little difference between control and roscovitine-treated nerve terminals during stimulation (Fig. $8 B, E, I$ ). This suggests that cdk5 activity does not play a role in the retrieval of single SVs from the plasma membrane. However, differences were apparent between control and roscovitine-treated nerve terminals with respect to the number of HRP-labeled SVs at time points after stimulation (Fig. 8I). The number of HRP-labeled SVs in

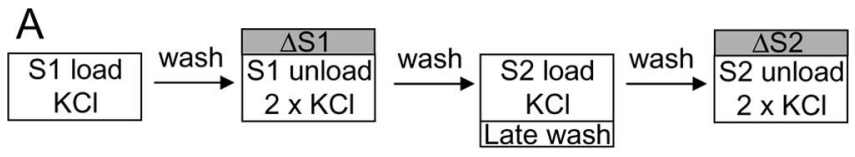

B

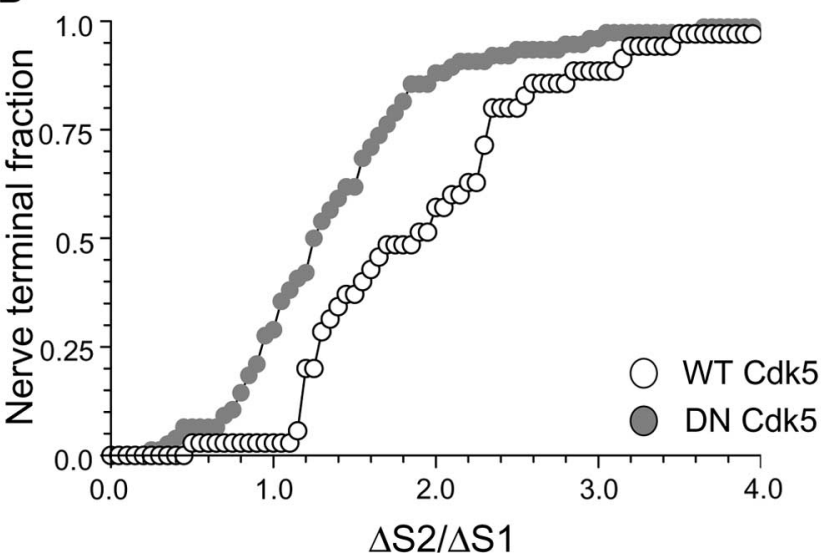

Figure 6. Overexpression of dominant-negative cdk5 inhibits the delayed uptake of FM210. $\boldsymbol{A}, \mathrm{FM2}-10$ was loaded using an identical protocol to Figure $1 A$ except wash of dye was delayed for 5 min after termination of stimulation at $\$ 2$. $B$, Cumulative histogram of the effect of either WT or DN cdk5 on dye loading in individual nerve terminals $(\Delta S 2 / \Delta S 1, n=35$, WT cdk5; $n=76$, DN cdk5). Open symbols, WT cdk5 transfected nerve terminals; gray symbols, DN cdk5-transfected nerve terminals. In both experiments, $n=3$.
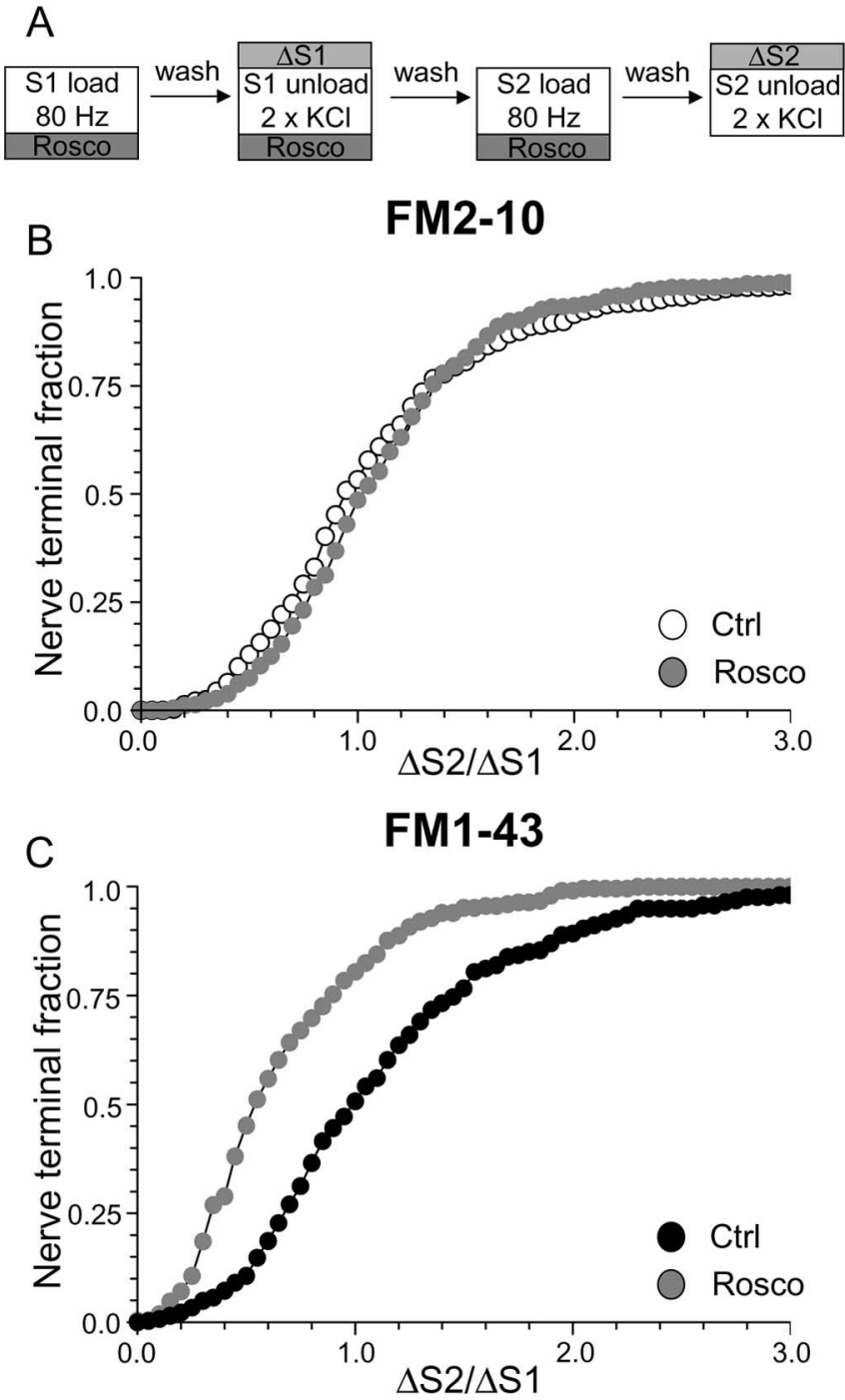

Figure 7. Antagonism of cdk5 inhibits uptake of FM1-43, but not FM2-10 during strong physiological stimulation. $\boldsymbol{A}$, Cultures were loaded with either FM2-10 or FM1-43 using 800 action potentials delivered at $80 \mathrm{~Hz}$. Dye was washed away immediately after stimulation. Unloading was stimulated with two sequential $30 \mathrm{~s}$ stimuli of $50 \mathrm{~mm} \mathrm{KCl}$. Cultures were preincubated with $50 \mu \mathrm{m}$ roscovitine (Rosco) for 10 min before and during all steps up to and including S2 loading. $\boldsymbol{B}, \boldsymbol{C}$, Cumulative histograms of the effect of roscovitine on either FM2-10 $(\boldsymbol{B})$ or FM1-43 (C) loading in individual nerve terminals are displayed $(\Delta \mathrm{S} 2 / \Delta \mathrm{S} 1, n=356$ control FM2-10; $n=141$, Rosco FM2-10; $n=262$, control FM1-43; $n=252$, Rosco FM143). Open symbols, FM2-10; closed symbols, FM1-43; gray symbols, roscovitine. For all experiments, $n=3$, except for $n=4$ for FM1-43 Rosco.

roscovitine-treated nerve terminals remained relatively constant, whereas in control nerve terminals, HRP-labeled SVs increased over time (Fig. 8I). The additional poststimulation HRP-labeled SVs must be generated from HRP-labeled endosomes, because HRP was washed away when stimulation terminated. Therefore, the inability of roscovitine-treated nerve terminals to generate HRP-labeled SVs after stimulation could either be caused by a lack of HRP-labeled endosomes from which these SVs can bud, or a direct effect on endosome budding itself. We hypothesize that the former possibility is most likely, because we found no difference in endosome diameters at later time points between roscovitine-treated and control nerve terminals (data not shown). Thus, inhibition of cdk5-dependent protein rephosphorylation selectively reduces the number of endosomes generated from the plasma membrane, whereas single SV endocytosis re- 


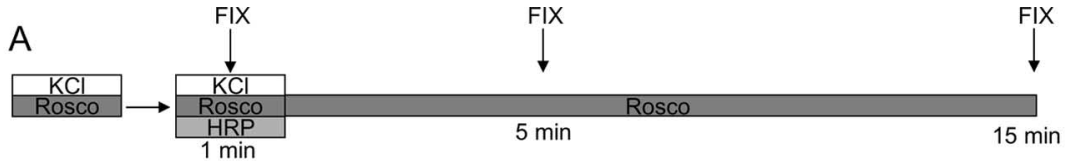

Ctrl

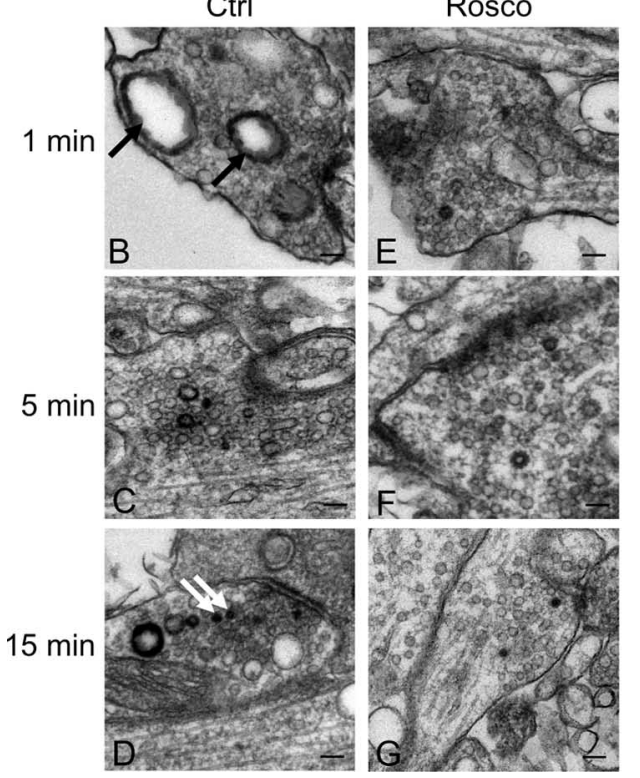

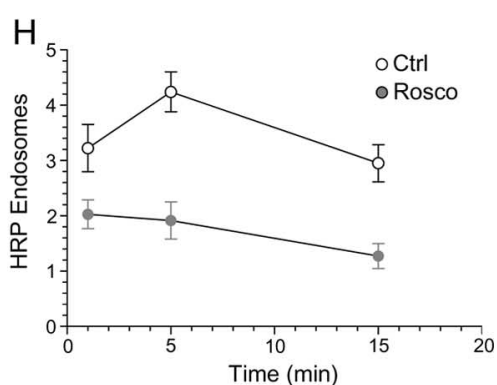

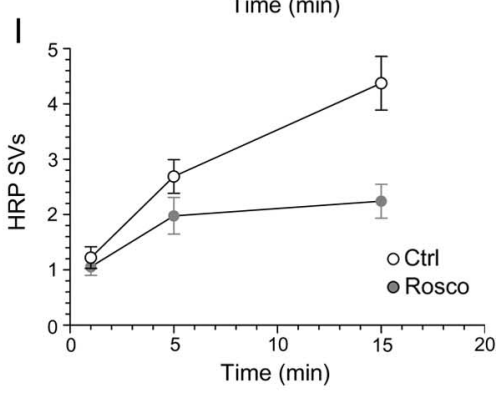

Figure 8. Inhibition of cdk5 selectively inhibits endosome generation and not single SV endocytosis. $A$, Cultures were stimulated for $10 \mathrm{~s}$ with $50 \mathrm{~mm} \mathrm{KCl}$, repolarized for $10 \mathrm{~min}$, and then stimulated again with $50 \mathrm{~mm} \mathrm{KCl}$ for $2 \mathrm{~min}$ in the presence of HRP. Cultures were repolarized for up to $15 \mathrm{~min}$ in the absence of HRP. Cultures were fixed at regular intervals during this time course and were preincubated with $50 \mu \mathrm{m}$ roscovitine (Rosco) for $10 \mathrm{~min}$ before the first $\mathrm{KCl}$ pulse and at all steps up to fixation where indicated. $\boldsymbol{B}-\boldsymbol{G}$, Representative electron micrographs of HRP uptake in control $(\boldsymbol{B}-\boldsymbol{D})$ or roscovitine-treated $(\boldsymbol{E}-\boldsymbol{G})$ nerve terminals at 1,5 , and 15 min after stimulation. Black arrows indicate HRP-labeled endosomal structures whereas white arrows indicate HRP-labeled SVs. Scale bars: $200 \mathrm{~nm}$. $\boldsymbol{H}$, Mean number of HRP-labeled endosomes per nerve terminal. I, Mean number of HRP-labeled SVs per nerve terminal. In $\boldsymbol{H}$ and $\boldsymbol{I}$, open circles represent control and closed circles represent roscovitine (Ctrl: $1 \mathrm{~min}$, $n=99$ nerve terminals; $5 \min , n=213 ; 15 \min , n=107 ;$ Rosco: $1 \mathrm{~min}, n=100 ; 5 \min , n=68 ; 15 \mathrm{~min}, n=43$ ). Error bars indicate SEM.

mains unaffected. This provides the morphological correlate for $\mathrm{cdk} 5$-dependent slow endocytosis, a generation of endosomal intermediates from which new SVs can bud.

\section{Activity-dependent requirement for cdk5 in SV endocytosis}

Slow endocytosis is reported to be activated by strong stimulation protocols, where the fast endocytic machinery becomes saturated by the number of SVs fusing with the nerve terminal plasma membrane (Richards et al., 2000). Thus, during mild stimulation, slow endocytosis should not be activated. To determine whether cdk5-dependent protein rephosphorylation was still required for SV endocytosis when challenged with a mild stimulus, the uptake of both FM1-43 and HRP was examined. We selected a loading protocol of 200 action potentials delivered at $10 \mathrm{~Hz}$ (Fig. $9 A$ ), which results in loading of $\sim 20 \%$ of the total recycling SV pool in our cultures (Evans, unpublished observations). Using this mild stimulation protocol, roscovitine did not inhibit FM1-43 loading, in contrast to its arrest of loading evoked by stronger stimulation (Fig. 9B). In fact, uptake of the dye was increased above control levels in the majority of nerve terminals (average $\Delta \mathrm{S} 2 / \Delta \mathrm{S} 1$ : control, $1.19 \pm 0.05 ;$ Rosco, $1.48 \pm 0.06 ; p<$ 0.05, two-way ANOVA, Bonferroni post hoc test). This was not caused by an increase in SV exocytosis because roscovitine did not increase FM1-43 unloading when present at S2 (data not shown). Thus, when nerve terminal stimulation is mild, SV endocytosis operates independently of cdk5 activity.

Because inhibition of cdk5 had no effect on single SV endocy- tosis during strong stimulation (Fig. 8), it is likely that the lack of effect of roscovitine on FM1-43 loading during mild stimulation was attributable to all retrieval events occurring via the single SV route. To test this hypothesis, we examined HRP uptake evoked by mild stimulation in our cultures. When this was performed, a selective labeling of single SVs was observed, with an almost complete absence of HRPlabeled endosomes (Fig. $10 B-D$ ). Therefore, during mild stimulation, membrane retrieval occurred almost exclusively via the single SV route, explaining the observed lack of effect of roscovitine on FM1-43 uptake.

To confirm that cdk5-dependent protein rephosphorylation was not required for single SV endocytosis during mild stimulation, HRP uptake in the presence of roscovitine was examined. As expected, roscovitine had no effect on the single SV route (Fig. 10E-G). Thus, cdk5dependent protein rephosphorylation does not control single SV endocytosis, which is the dominant pathway during mild stimulation. Instead, it recruits an additional slow endocytosis pathway during strong stimulation that traffics SV membrane via an endosomal route. Thus, the phosphorylation status of the dephosphins controls SV endocytosis in an activity-dependent manner by recruiting an additional slow endosomal pathway during strong stimulation.

\section{SVs generated by slow endocytosis in nerve terminals allow sustained release}

SVs generated via slow endocytosis are only released after fusion of SVs generated via a fast endocytic route in peripheral synapses (Richards et al., 2000). Thus, we would predict that inhibition of the slow endocytic pathway would result in inhibition of a sustained phase of release during strong stimulation. We tested this hypothesis by examining two different kinetic components of SV unloading after loading dye with strong stimulation. First, we determined the time taken for either FM2-10- or FM1-43loaded nerve terminals to lose the initial $50 \%$ of their dye content at $\mathrm{S} 1$ (Fig. $11 A, \mathrm{t}_{1 / 2}$ ). We found no difference between the initial rate of dye unloading between FM2-10 and FM1-43, suggesting equal labeling of the single SV pathway (FM2-10, $4.24 \pm 0.37 \mathrm{~s}$; FM1-43, $5.3 \pm 0.44 \mathrm{~s} ; n=14$ experiments \pm SEM; $p=0.76$, Student's $t$ test). Second, we determined the rate of unloading for the remaining dye, because this may include SVs generated by slow endocytosis and, thus, selectively labeled by FM1-43. We found that unloading of FM1-43 was sustained for significantly longer than FM2-10 (FM2-10, $13.95 \pm$ 1.05; FM1-43, $19.56 \pm$ $1.24 ; n=14$ experiments \pm SEM; $p=0.002$, Student's $t$ test) indicating a selective labeling of a slowly recycling pool of SVs by FM1-43.

Because the sustained phase of FM1-43 unloading probably originates from SVs generated via slow endocytosis, we predicted that inhibition of either calcineurin or $c \mathrm{dk} 5$ should selectively eliminate this phase of release. The presence of either CsA or 


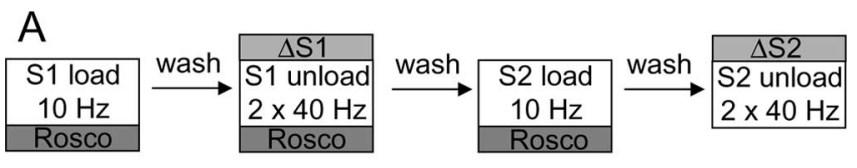

B FM1-43

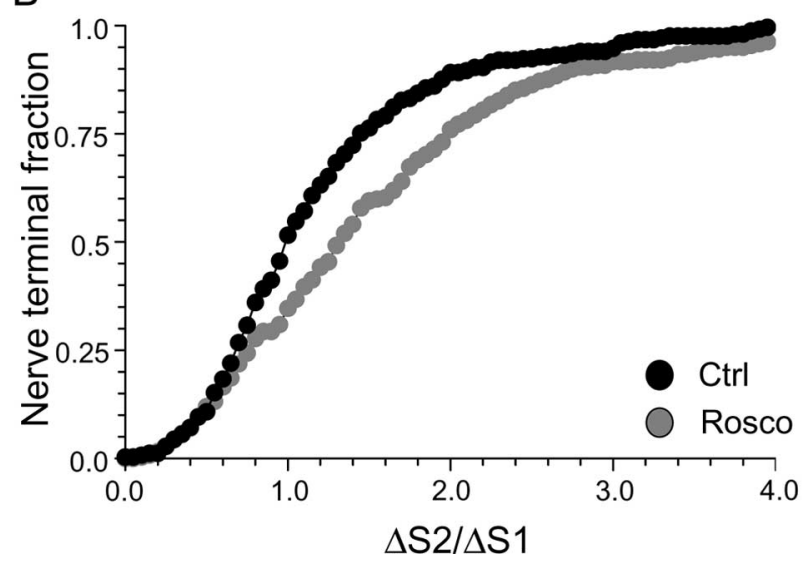

Figure 9. C $\mathrm{dk} 5$ is not required for endocytosis evoked by mild stimulation. $A$, FM1- 43 was loaded using 200 action potentials delivered at $10 \mathrm{~Hz}$ and immediately washed away after termination of stimulation. Dye unloading was evoked by two sequential stimuli of 400 action potentials delivered at $40 \mathrm{~Hz}$. Cultures were preincubated with $50 \mu \mathrm{m}$ roscovitine (Rosco) for 10 min before and during all steps up to and including $\$ 2$ loading. $\boldsymbol{B}$, Cumulative histogram of the effect of roscovitine on dye loading in individual nerve terminals ( $\Delta S 2 / \Delta S 1, n=250, \mathrm{Ctrl} ; n=$ 322, Rosco). Closed symbols represent control (Ctrl) and gray symbols represent roscovitine. In all experiments, $n=3$ for control and $n=4$ for roscovitine.

roscovitine during S2 loading had no effect on the initial kinetics of dye unloading $\left(t_{1 / 2}\right)$ for either FM2-10 or FM1-43 (Fig. $11 B$ ). This was expected because the single SV endocytosis route is unaffected by antagonism of either calcineurin or cdk5. However, both drugs eliminated the sustained unloading of FM1-43, while not affecting the residual unloading of FM2-10 (Fig. 11C). This shows that inhibition of slow endocytosis results in depletion of a slowly recycling pool of SVs and that replenishment of this pool is dependent on both calcineurin and cdk5 activity.

\section{Discussion}

We have shown that ablation of either calcineurin or cdk5 activity controls a slow form of endocytosis in central nerve terminals that requires traffic via endosomal intermediates. Inhibition of protein dephosphorylation by calcineurin or protein rephosphorylation by cdk5 arrests the uptake of FM1-43 but not FM2-10, suggesting control of an endocytic pathway selectively labeled by the former, more hydrophobic, dye. This was confirmed by experiments that showed that FM2-10 uptake was also dependent on these enzymes when its washout was delayed after the loading stimulus. We have identified a morphological correlate for this pathway because inhibition of cdk5 activity reduced endosome, but not SV, generation from the plasma membrane during strong stimulation. The requirement for cdk5-dependent protein rephosphorylation was activity-dependent, because cdk5 inhibition did not affect SV endocytosis evoked by mild stimulation protocols, which exclusively use the single SV route. Together, these data reveal for the first time the specific SV endocytosis pathway that is regulated by calcineurin-dependent dephosphorylation and cdk5-dependent protein rephosphorylation and, thus, by extension, the phosphorylation cycle of the dephosphins.

The technique of using styryl dyes of differing hydrophobicity to identify separate endocytosis pathways was first used in peripheral synapses (Richards et al., 2000, 2004). FM1-43 labeled an additional endocytic pathway to FM2-10 during tetanic stimulation, which was identified as a pathway involving endosomes/ cisternae from which new SVs could bud (Richards et al., 2000). We propose that the slow endocytosis pathway revealed in our cultures using FM2-10 and FM1-43 is related to that observed in peripheral synapses because they follow the same criteria seen for peripheral synapses. One of these criteria is that when the washout of FM2-10 was delayed after termination of the loading stimulus, the slow endocytosis pathway was labeled. This additional loading is not caused by spontaneous or asynchronous SV turnover because this dye can be unloaded by a depolarizing stimulus (Sara et al., 2005). In contrast, no additional loading of FM1-43 is observed when its washout is delayed, when compared with its immediate washout after termination of stimulation (M. A. Cousin, unpublished observation). This is important because it shows that FM1-43 can label slowly retrieving structures even after its washout.

In central synapses, FM2-10 and FM1-43 have also been used in concert, however mostly in attempts to identify different modes of SV fusion (e.g., kiss-and-run vs full fusion) (Ryan et al., 1996; Klingauf et al., 1998; Pyle et al., 2000; Stevens and Williams, 2000). The hypothesis formed in these studies was that, because the dyes had different departition rates from membrane, the more hydrophobic dyes would not fully escape SVs in cases where transient fusion with the plasma membrane occurred, such as kiss-and-run. This appeared to be the case in a number of studies where more FM2-10 was unloaded in comparison to FM1-43 for the same stimulus (Klingauf et al., 1998; Pyle et al., 2000). However, not all studies demonstrated such a disparity (Ryan et al., 1996; Stevens and Williams, 2000) and it became apparent that the key event in obtaining differential dye release was not the unloading stimulus at all, but in fact the loading stimulus. In instances in which a mild loading stimulus was used, no disparity in dye unloading was observed (Ryan et al., 1996; Stevens and Williams, 2000) whereas a strong loading stimulus such as $\mathrm{KCl}$ resulted in observed disparity on unloading (Klingauf et al., 1998; Pyle et al., 2000). This resulted from FM1-43 labeling an additional pool of SVs, some of which were trafficking via the endosomal route and thus were not immediately available for unloading as observed in this manuscript (Fig. 11). Thus, FM2-10 and FM1-43 in concert can offer insights into the mode of SV retrieval but not SV fusion states (Virmani et al., 2003).

\section{Molecular mechanism of slow endocytosis}

Previous morphological studies using either photoconversion of FM dyes in peripheral nerve terminals (Richards et al., 2000, 2004) or HRP uptake in central nerve terminals (Marxen et al., 1999; Leenders et al., 2002; de Lange et al., 2003; Di Paolo et al., 2004) have suggested that the slow endocytosis pathway is morphologically distinct from single SV endocytosis, with label accumulated in large endosomes or cisternae in addition to SVs. Interestingly, no labeling of this slow pathway has been observed using photoconversion of FM dyes in central nerve terminals regardless of stimulation protocol (Harata et al., 2001). This is probably because of the inability of dyes to be converted when inside round endosomes (de Lange et al., 2003). SVs are derived from these cisternal structures, because in our study and in others HRP endosomes disappear and HRP-labeled SV increase over time after extracellular HRP and stimulation have been removed (Marxen et al., 1999; Leenders et al., 2002; de Lange et al., 2003). However, the mechanism by which large endosomes are gener- 
A
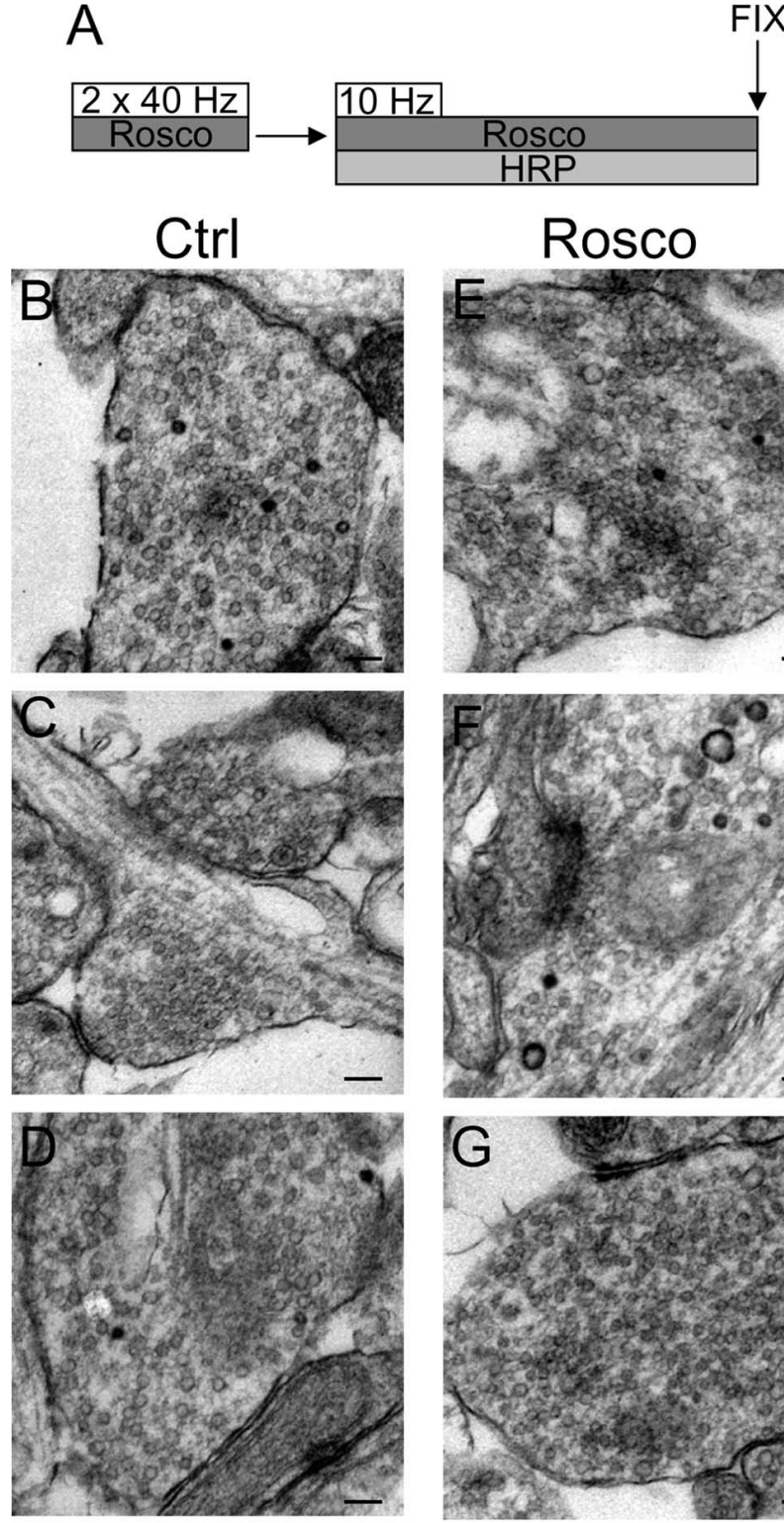

$\mathrm{H}_{20}$

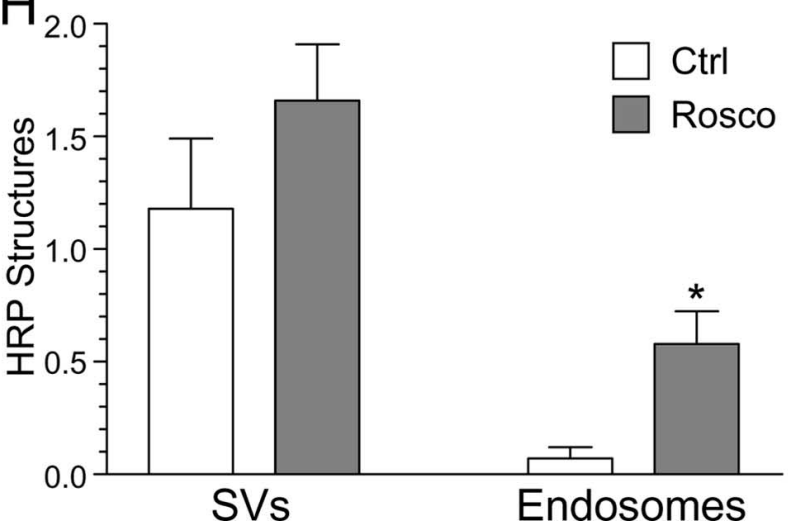

Figure 10. Mild stimulation evokes a specific single SV endocytosis pathway independent of cdk5 activity. $A$, Cultures were stimulated twice for $10 \mathrm{~s}$ with two trains of 400 action potentials $(40 \mathrm{~Hz})$, repolarized for $10 \mathrm{~min}$, and then stimulated again with a train of 200 action potentials $(10 \mathrm{~Hz})$ in the presence of HRP. HRP was kept present for an additional 60 s to ensure labeling of all retrieval pathways and then cultures were fixed. Cultures were preincubated with $50 \mu \mathrm{m}$ roscovitine (Rosco) for $10 \mathrm{~min}$ before the first stimulations and at all steps up to fixation where indicated. $\boldsymbol{B}-\boldsymbol{G}$, Representative electron micrographs of HRP uptake in control $(\boldsymbol{B}-\boldsymbol{D})$ or roscovitine-treated $(\boldsymbol{E}-\boldsymbol{G})$ nerve terminals. Scale bars, $200 \mathrm{~nm}$. Five roscovitine nerve terminals
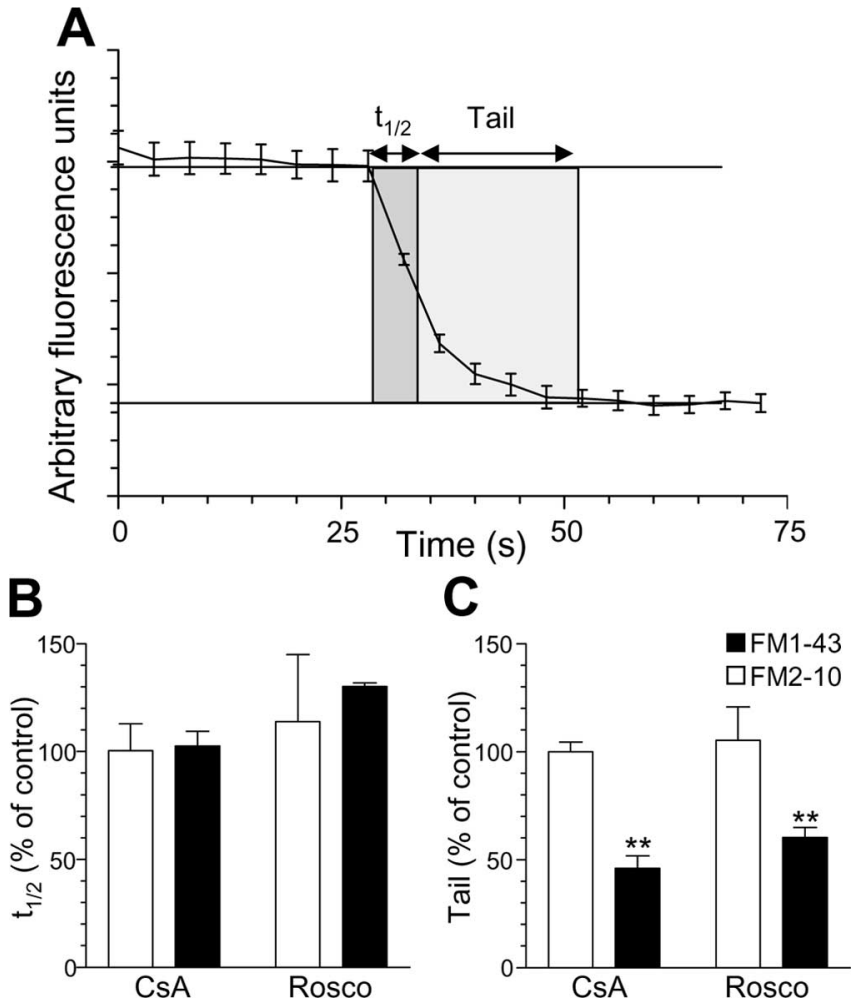

Figure 11. Inhibition of slow endocytosis results in elimination of a sustained phase of SV exocytosis. $\boldsymbol{A}$, Two parameters were monitored with respect to dye unloading, the time for nerve terminals to lose $50 \%$ of their dye content $\left(\mathrm{t}_{1 / 2}\right.$, shown in dark gray) and the time for the remainder to be unloaded (Tail, shown in light gray). In all experiments, dye was loaded with 50 $\mathrm{mm} \mathrm{KCl}$ and washed away immediately after stimulation as in Figure $1 \mathrm{~A}$. Dye was unloaded with $50 \mathrm{~mm} \mathrm{KCl}$. Cultures were preincubated with either $10 \mu \mathrm{m}$ CsA before and during S2 loading or $50 \mu \mathrm{m}$ Rosco at all steps including S2 loading. B, Effect of CSA and roscovitine (Rosco) on the time taken to unload the initial $50 \%$ of dye content $\left(\mathrm{t}_{1 / 2}\right)$. C, Effect of CsA and Rosco on the time taken to unload the remainder of dye (Tail). Data are presented as either $t_{1 / 2}$ or tail kinetics at $S 2$ normalized to the internal $S 1$ control ( $n=6$ experiments for FM2-10 CsA; $n=3$ for FM2-10 Rosco; $n=4$ for FM1- 43 CsA; $n=3$ for FM1- 43 Rosco; all data are \pm SEM; Student's $t$ test, $\left.{ }^{* *} p<0.01\right)$.

ated is still unclear. Such structures could either originate from the fusion of HRP-labeled SVs with a sorting endosome (Heuser and Reese, 1973) or from bulk invagination of the plasma membrane in a process similar to macropinocytosis (Gad et al., 1998; Richards et al., 2000, 2004; Teng and Wilkinson, 2000; Holt et al., 2003). Inhibition of either process would result in our observed phenotype on ablation of cdk5 activity, a reduction in the numbers of HRP-labeled endosomes in nerve terminals. Importantly, for this study we have shown that slow endocytosis is not the same process that lags behind because of saturation of the endocytic machinery, but rather a process that has, if not a different molecular mechanism, at least a different regulatory mechanism to fast endocytosis.

It is most likely that fast and slow endocytosis have a very similar molecular mechanism and only differ in their regulation. Thus, the phosphorylation cycle of the dephosphins may control a slow endocytic pathway that has some alternative molecular

$\leftarrow$

were discounted because they had an atypical number of HRP endosomes compared with the rest (total $=57)$. $\boldsymbol{H}$, Mean number of HRP-labeled structures per nerve terminal for either control (open bars) or roscovitine treated (gray bars) cultures \pm SEM (Ctrl, $n=29 ;$ Rosco, $n=$ $51 ;{ }^{*} p<0.05$, Student's $t$ test). 
players to fast endocytosis. In support, phosphorylation of the proline-rich $\mathrm{C}$ terminus of dynamin I regulates interactions with its major binding partners amphiphysin, endophilin, and syndapin to widely differing extents (Anggono et al., 2006). It would be plausible to propose that these proteins [all containing dynamin-binding SH3 domains and either N-Bin/amphiphysin/ RVS (BAR) or F-BAR lipid binding domains] (Peter et al., 2004; Itoh et al., 2005) may perform similar roles in distinct but related endocytic pathways. We hypothesize that the dynamin I-syndapin I interaction may be critical for slow endocytosis, because this interaction is completely dependent on the phosphorylation status of dynamin I (Anggono et al., 2006).

\section{The dephosphins are activity-dependent sensors for slow endocytosis}

Activity-dependent slow endocytosis has been revealed recently using the SV protein synaptophysin as a membrane tracker (Granseth et al., 2006). When hippocampal cultures were challenged with very mild stimulation (up to 40 action potentials), only one form of endocytosis was apparent that had a time constant of $15 \mathrm{~s}$. However, when stimulation intensity was raised above this level, endocytosis occurred with a new time constant of $50 \mathrm{~s}$ (Granseth et al., 2006). We propose that the phosphorylation status of the dephosphins controls a slow endocytic pathway that uses endosomal intermediates during strong stimulation. Our data suggest that rather than slow endocytosis being automatically triggered by the engulfment of the endocytic machinery, it is activated by the dephosphorylation of the dephosphins. In support, SV turnover in hippocampal neurones was only dependent on calcineurin activity when strong stimulation protocols were used (Kumashiro et al., 2005). Also, in chromaffin cells, endocytosis evoked by strong physiological stimulation is calcineurindependent, but endocytosis evoked by mild stimulation was not (Chan and Smith, 2001). The dephosphorylation of the dephosphins should be the ideal sensor for the stimulation of slow endocytosis because only strong stimulation will elevate intracellular calcium high enough for calcineurin to dephosphorylate these cytosolic proteins outside the active zone. In agreement, we observe a robust frequency-dependent dephosphorylation of dynamin I in our culture system (Cousin, unpublished observation) that closely correlates with the stimulation of slow endocytosis. Thus, we propose that slow endocytosis is stimulated by changes in the phosphorylation status of the dephosphins and that saturation of the fast endocytic machinery is simply a consequence of strong stimulation rather than the trigger itself.

In summary, we have identified the calcineurin- and cdk5mediated phosphorylation cycle of the dephosphins as the activity-dependent trigger for slow endocytosis in central nerve terminals. The next challenge will be to identify how the phosphorylation status of individual dephosphins controls this endocytic pathway.

\section{References}

Anggono V, Smillie KJ, Graham ME, Valova VA, Cousin MA, Robinson PJ (2006) Syndapin I is the phosphorylation-regulated dynamin I partner in synaptic vesicle endocytosis. Nat Neurosci 9:752-760.

Aravanis AM, Pyle JL, Tsien RW (2003) Single synaptic vesicles fusing transiently and successively without loss of identity. Nature 423:643-647.

Chan SA, Smith C (2001) Physiological stimuli evoke two forms of endocytosis in bovine chromaffin cells. J Physiol (Lond) 537:871-885.

Cousin MA (2000) Synaptic vesicle endocytosis: calcium works overtime in the nerve terminal. Mol Neurobiol 22:115-128.

Cousin MA, Robinson PJ (2001) The dephosphins: dephosphorylation by calcineurin triggers synaptic vesicle endocytosis. Trends Neurosci 24:659-665.
Cousin MA, Tan TC, Robinson PJ (2001) Protein phosphorylation is required for endocytosis in nerve terminals. Potential role for the dephosphins dynamin I and synaptojanin, but not AP180 or amphiphysin. J Neurochem 76:105-116.

Deak F, Schoch S, Liu X, Sudhof TC, Kavalali ET (2004) Synaptobrevin is essential for fast synaptic-vesicle endocytosis. Nat Cell Biol 6:1102-1108.

de Lange RP, de Roos AD, Borst JG (2003) Two modes of vesicle recycling in the rat calyx of Held. J Neurosci 23:10164-10173.

Di Paolo G, Moskowitz HS, Gipson K, Wenk MR, Voronov S, Obayashi M, Flavell R, Fitzsimonds RM, Ryan TA, De Camilli P (2004) Impaired PtdIns $(4,5) \mathrm{P}_{2}$ synthesis in nerve terminals produces defects in synaptic vesicle trafficking. Nature 431:415-422.

Gad H, Low P, Zotova E, Brodin L, Shupliakov O (1998) Dissociation between $\mathrm{Ca}^{2+}$-triggered synaptic vesicle exocytosis and clathrin-mediated endocytosis at a central synapse. Neuron 21:607-616.

Gandhi SP, Stevens CF (2003) Three modes of synaptic vesicular recycling revealed by single-vesicle imaging. Nature 423:607-613.

Granseth B, Odermatt B, Royle SJ, Lagnado L (2006) Clathrin-mediated endocytosis is the dominant mechanism of vesicle retrieval at hippocampal synapses. Neuron 51:773-786.

Harata N, Ryan TA, Smith SJ, Buchanan J, Tsien RW (2001) Visualizing recycling synaptic vesicles in hippocampal neurons by FM 1-43 photoconversion. Proc Natl Acad Sci USA 98:12748-12753.

Harata NC, Choi S, Pyle JL, Aravanis AM, Tsien RW (2006) Frequencydependent kinetics and prevalence of kiss-and-run and reuse at hippocampal synapses studied with novel quenching methods. Neuron 49:243-256.

Heuser JE, Reese TS (1973) Evidence for recycling of synaptic vesicle membrane during transmitter release at the frog neuromuscular junction. J Cell Biol 57:315-344.

Holt M, Cooke A, Wu MM, Lagnado L (2003) Bulk membrane retrieval in the synaptic terminal of retinal bipolar cells. J Neurosci 23:1329-1339.

Itoh T, Erdmann KS, Roux A, Habermann B, Werner H, De Camilli P (2005) Dynamin and the actin cytoskeleton cooperatively regulate plasma membrane invagination by BAR and F-BAR proteins. Dev Cell 9:791-804.

Klingauf J, Kavalali ET, Tsien RW (1998) Kinetics and regulation of fast endocytosis at hippocampal synapses. Nature 394:581-585.

Koenig JH, Ikeda K (1996) Synaptic vesicles have two distinct recycling pathways. J Cell Biol 135:797-808.

Kumashiro S, Lu YF, Tomizawa K, Matsushita M, Wei FY, Matsui H (2005) Regulation of synaptic vesicle recycling by calcineurin in different vesicle pools. Neurosci Res 51:435-443.

Lee SY, Wenk MR, Kim Y, Nairn AC, De Camilli P (2004) Regulation of synaptojanin 1 by cyclin-dependent kinase 5 at synapses. Proc Natl Acad Sci USA 101:546-551.

Lee SY, Voronov S, Letinic K, Nairn AC, Di Paolo G, De Camilli P (2005) Regulation of the interaction between PIPKI gamma and talin by prolinedirected protein kinases. J Cell Biol 168:789-799.

Leenders AG, Scholten G, de Lange RP, Lopes Da Silva FH, Ghijsen WE (2002) Sequential changes in synaptic vesicle pools and endosome-like organelles during depolarization near the active zone of central nerve terminals. Neuroscience 109:195-206.

Liu JP, Sim ATR, Robinson PJ (1994) Calcineurin inhibition of dynamin I GTPase activity coupled to nerve terminal depolarization. Science 265:970-973.

Marks B, McMahon HT (1998) Calcium triggers calcineurin-dependent synaptic vesicle recycling in mammalian nerve terminals. Curr Biol 8:740-749.

Marxen M, Volknandt W, Zimmermann H (1999) Endocytic vacuoles formed following a short pulse of $\mathrm{K}^{+}$-stimulation contain a plethora of presynaptic membrane proteins. Neuroscience 94:985-996.

Murthy VN, Stevens CF (1998) Synaptic vesicles retain their identity through the endocytic cycle. Nature 392:497-501.

Peter BJ, Kent HM, Mills IG, Vallis Y, Butler PJ, Evans PR, McMahon HT (2004) BAR domains as sensors of membrane curvature: the amphiphysin BAR structure. Science 303:495-499.

Pyle JL, Kavalali ET, Piedras-Renteria ES, Tsien RW (2000) Rapid reuse of readily releasable pool vesicles at hippocampal synapses. Neuron 28:221-231.

Richards DA, Guatimosim C, Betz WJ (2000) Two endocytic recycling routes selectively fill two vesicle pools in frog motor nerve terminals. Neuron 27:551-559. 
Richards DA, Guatimosim C, Rizzoli SO, Betz WJ (2003) Synaptic vesicle pools at the frog neuromuscular junction. Neuron 39:529-541.

Richards DA, Rizzoli SO, Betz WJ (2004) Effects of wortmannin and latrunculin A on slow endocytosis at the frog neuromuscular junction. J Physiol (Lond) 557:77-91.

Rizzo MA, Springer GH, Granada B, Piston DW (2004) An improved cyan fluorescent protein variant useful for FRET. Nat Biotechnol 22:445-449.

Rizzoli SO, Betz WJ (2005) Synaptic vesicle pools. Nat Rev Neurosci 6:57-69.

Ryan TA, Smith SJ, Reuter H (1996) The timing of synaptic vesicle endocytosis. Proc Natl Acad Sci USA 93:5567-5571.

Sara Y, Virmani T, Deak F, Liu X, Kavalali ET (2005) An isolated pool of vesicles recycles at rest and drives spontaneous neurotransmission. Neuron 45:563-573.

Smillie KJ, Evans GJ, Cousin MA (2005) Developmental change in the calcium sensor for synaptic vesicle endocytosis in central nerve terminals. J Neurochem 94:452-458.

Stevens CF, Williams JH (2000) "Kiss and run" exocytosis at hippocampal synapses. Proc Natl Acad Sci USA 97:12828-12833.
Takei K, Mundigl O, Daniell L, De Camilli P (1996) The synaptic vesicle cycle: a single vesicle budding step involving clathrin and dynamin. J Cell Biol 133:1237-1250.

Tan TC, Valova VA, Malladi CS, Graham ME, Berven LA, Jupp OJ, Hansra G, McClure SJ, Sarcevic B, Boadle RA, Larsen MR, Cousin MA, Robinson PJ (2003) Cdk5 is essential for synaptic vesicle endocytosis. Nat Cell Biol 5:701-710

Teng H, Wilkinson RS (2000) Clathrin-mediated endocytosis near active zones in snake motor boutons. J Neurosci 20:7986-7993.

Vanden Berghe P, Klingauf J (2006) Synaptic vesicles in hippocampal boutons recycle to different pools in a use-dependent fashion. J Physiol (Lond) 572:707-720.

Virmani T, Han W, Liu X, Sudhof TC, Kavalali ET (2003) Synaptotagmin 7 splice variants differentially regulate synaptic vesicle recycling. EMBO J 22:5347-5357.

Yan Z, Chi P, Bibb JA, Ryan TA, Greengard P (2002) Roscovitine: a novel regulator of $\mathrm{P} / \mathrm{Q}$-type calcium channels and transmitter release in central neurons. J Physiol (Lond) 540:761-770. 